Sociologie et sociétés

\title{
Le credo identitaire comme ressource pour l'art ?
}

\author{
L'exemple français de la région toulousaine
}

The Identity Credo as a Resource for Art ?

The French Case of the Toulouse Region

\section{Martine Azam}

Volume 34, numéro 2, automne 2002

Les territoires de l'art

Art Territories

URI : https://id.erudit.org/iderudit/008138ar

DOI : https://doi.org/10.7202/008138ar

Aller au sommaire du numéro

Éditeur(s)

Les Presses de l'Université de Montréal

ISSN

0038-030X (imprimé)

1492-1375 (numérique)

Découvrir la revue

Citer cet article

Azam, M. (2002). Le credo identitaire comme ressource pour l'art ? L'exemple français de la région toulousaine. Sociologie et sociétés, 34(2), 185-205.

https://doi.org/10.7202/008138ar

\section{Résumé de l'article}

À travers un siècle de relations entre art et territoire en région Midi-Pyrénées, l'article s'intéresse à la façon dont les acteurs, collectifs et individuels, ont érigé en ressources les notions de territoire et d'identité. Abordée de façon chronologique, la première partie de l'article retrace les périodes au cours desquelles le mouvement régionaliste et les milieux artistiques se sont retrouvés pour porter la revendication d'une identité spécifique. La seconde partie cherche à dépasser les circonstances propres à chacun des moments de l'histoire régionale et dégage les composantes humaines et discursives - les acteurs, le discours identitaire, le « retard » — et les variantes qu'elles enregistrent. 


\section{Le credo identitaire comme ressource pour l'art?}

L'exemple français de la région toulousaine

\section{MARTINE AZAM}

CERS, UMR 5117

Maison de la recherche

5, Allées Antonio Machado

31058 Toulouse Cedex, France

Courriel : martine.azam@univ-tlse2.fr

$\mathrm{E}$

N FRANCE, LES ARTS PLASTIQUES ONT REPRÉSENTÉ, pour les provinces d'abord et pour les régions ensuite, une arme durant les périodes d'opposition à la centralité parisienne. Ce trait est particulièrement saillant dans la région Midi-Pyrénées qui revendique une identité culturelle forte, incarnée par «Toulouse, capitale historique du Languedoc». L'existence d'un débat sur les conditions d'exercice et de reconnaissance d'un art produit localement n'est pas nouveau en Midi-Pyrénées: il se décline dans des termes proches chaque fois que réapparaît l'opposition à la centralité parisienne et il se trouve réactualisé à l'heure où les particularités sont portées par la protestation antimondialisation.

Les tenants d'un art contemporain, forcément international, ne cessent de rappeler la nécessaire dimension universelle de l'art, laquelle s'oppose dans sa logique même à toute localisation. Ils mettent en garde contre toute revendication identitaire qu'ils identifient à une attitude de repli et, dans ce registre, la difficulté de la région à enfanter de «grands artistes» serait liée à un localisme servant d'alibi à la médiocrité. Selon cette logique, la région Midi-Pyrénées n'aurait finalement que ce qu’elle mérite et, dans un paysage artistique sans frontières, l'art qui se fait localement est assimilé à un art local, avec la dimension péjorative attachée à ce terme.

Les tenants d'un art contemporain respectueux des spécificités culturelles prônent la mise en valeur d'une expression artistique qui trouverait dans le local une tonalité par- 
ticulière. Ils mettent en accusation l'uniformisation des expressions artistiques dont le label de contemporanéité servirait à masquer la domination d'une élite internationale à forte coloration anglo-saxonne. Dans cette vision, les artistes régionaux qui cherchent à se situer dans ce concert international sont, au mieux, des suiveurs réduits à occuper des strapontins, et les institutions et galeries régionales sont les succursales d'un art élaboré ailleurs et à l'intention de quelques privilégiés. Alimenté, notamment, par la recherche sociologique, ce débat s'appuie en toile de fond sur l'idée, largement répandue par les combats régionalistes depuis le début du siècle, qu'un territoire est producteur d'une identité culturelle commune aux individus qui partagent un même espace et une histoire spécifique.

La réflexion que nous proposons ici n'a pas pour objet d'examiner le bien-fondé des arguments déployés par l'un et l'autre camps, ni d'évaluer la solidité des faits dont ils se nourrissent. Inscrite dans la position constructiviste, elle cherche à saisir la façon dont les acteurs collectifs et individuels érigent en ressources les notions de territoire et d'identité. En effet, ces notions sont au cœur même de l'idée de région telle qu'elle est définie par P. Lagarde: "une communauté stable, historiquement constituée, de langue, de territoire, de vie économique et de formation psychique, qui se traduit par une communauté de culture» (Lagarde, 1977, p. 76). Posée ainsi, cette définition fait oublier que la région résulte justement d'une construction collective à dimension historique et ce terme ne serait rien sans celui de «régionalisme» qui lui donne consistance et renvoie explicitement au travail des acteurs pour faire exister une ou plusieurs conceptions parfois contradictoires de cette identité. Identité, territoire, ces notions ne sont pas définies une fois pour toutes: à chaque étape du mouvement historique, elles s'affirment dans des réalités sociales nouvelles et toujours mouvantes. À ce titre, l'exemple midi-pyrénéen des relations entre arts plastiques, territoire et identité, peut nourrir une réflexion sur l'articulation problématique entre le crédo identitaire et celui de la contemporanéité esthétique.

Quel sort les acteurs de Midi-Pyrénées ont-ils réservé au discours identitaire? Plus précisément, dans quelles circonstances les différents participants du monde de l'art régional ont-ils mobilisé et décliné cette thématique et avec quels effets sur le monde de l'art régional? Quels liens peut-on établir entre l'absence de visibilité de la région en matière d'arts plastiques et la revendication d'une identité régionale?

La première partie de l'article propose de répondre à ces questions par une approche sociohistorique qui montre que la question régionale, et avec elle la question identitaire, sert des intérêts divers, prend diverses nuances en fonction des groupes d'acteurs qui la posent et du moment où ils la posent. La période présentée couvre le $\mathrm{xx}^{\mathrm{e}}$ siècle, allant de la fin des années 1890, période à laquelle naît l'idée d'une «école de peinture toulousaine», à la fin des années 1990 qui voit définitivement assise l'existence d'un «monde de l'art» contemporain. Ainsi, sur ce siècle qui a donné au paysage artistique régional sa configuration actuelle, nous pointerons les périodes au cours desquelles le mouvement régionaliste et les milieux artistiques se sont retrouvés pour porter la revendication d'une identité spécifique. Nous verrons comment, à la méri- 
dionalité nostalgique du début du $\mathrm{xx}^{\mathrm{e}}$ siècle succède dans les années 1950 un occitanisme en quête de modernité qui pose les bases des revendications régionalistes des années 1970; enfin, avec la décentralisation des années 1980, c'est à l'absorption progressive de la thématique identitaire que l'on assiste.

En comparant ces différents moments de l'histoire régionale, nous chercherons dans un deuxième temps à mettre en évidence les composantes humaines et discursives de la thématique identitaire. Entre les principales catégories d'acteurs, nous verrons qui, des édiles ou des artistes, «mène la danse». Nous suivrons également, à travers les tentatives successives pour faire exister une «École toulousaine», les participants au monde de l'art dans leur difficulté à donner une substance proprement plastique à l'idée d'identité culturelle. Enfin, ces composantes humaines et discursives de la thématique identitaire conduiront à examiner une idée insistante qui a cours dans les milieux artistiques locaux et nationaux: celle d'un «retard» de Midi-Pyrénées en matière d'arts plastiques.

Les analyses qui suivent s'appuient sur les résultats de deux recherches existantes qu'elles réinterrogent: celle consacrée à la vie artistique locale jusqu'au début de la Seconde Guerre mondiale, par L. Rivet, historienne d'art (Rivet, 1989); celle conduite par nos soins pour la période couvrant la deuxième partie du siècle (Azam, 1998). Cette dernière a utilisé les matériaux traditionnels en histoire-dépouillement d'archives et documents multiples - et complété les investigations par les éléments tirés d'une quarantaine d'entretiens de témoins et d'acteurs de la vie artistique locale.

\section{UNE HISTOIRE DU CRÉDO IDENTITAIRE}

\section{Première période: la méridionalité comme repli}

Toulouse est pour le Sud-Ouest un pôle particulièrement important: ville universitaire de tradition juridique, de culture occitane, elle a une vie intellectuelle importante et compte de nombreuses sociétés savantes. Mais au début du siècle, le mouvement méridional du Sud-Ouest et plus particulièrement de la région toulousaine est en retrait des actions menées dans le Sud-Est par Le Félibrige $e^{1}$ qui milite pour une reconnaissance occitane. À Toulouse, la revendication d'une identité propre s'ancre surtout dans un refus du pouvoir central. Portée par une gauche de notables, ses composantes rejoignent celles du mouvement félibréen tel que l'analyse R. Lafont (Lafont, 1974). La capitale régionale devient, à la fin du XIX $^{e}$ siècle, le bastion d'une gauche qui entend bien résister aux empiètements du centralisme parisien. À la faveur du remodelage du tissu urbain, les arts plastiques deviennent un moyen de propagande, autant à destination de la population locale qu'à celle du pouvoir central. Cela se manifeste particulièrement

1. Le Félibrige est un mouvement littéraire né au $\mathrm{XIX}^{\mathrm{e}}$ siècle en Provence qui se caractérise par l'abondance particulière des écrivains et des publications, et la création d'institutions culturelles. Ce mouvement s'étend dans le couloir du Rhône et entre Béziers et Marseille. Il est animé par les poètes d'Arles et d'Avignon dont la figure de proue est l'écrivain Frédéric Mistral. Le Félibrige finit avec la mort de Mistral et la guerre en 1914. 
lorsque la décoration de la salle des Illustres de l'hôtel de ville de Toulouse est entreprise. Les choix du pouvoir central participant aux coûts d'embellissement se heurtent à ceux des élus locaux soucieux de promouvoir des artistes régionaux. On assiste alors à une véritable mobilisation de toutes les catégories d'acteurs locaux concernés. Parmi eux, les politiques bien sûr, mais également les artistes d'origine toulousaine qui, collectivement, proposent d'assurer la décoration de l'édifice public ${ }^{2}$. C'est pendant cette période de désaccord avec l'État (entre 1888 et 1894) que naît l'idée d'une «école toulousaine» de peinture. Pour L. Rivet (Rivet, 1989), il s'agit là d'un éclectisme académique qui réunit des maîtres locaux et «ces grands toulousains de Paris »3. L'attachement de ces derniers à leur origine est lié en partie à un sentiment de reconnaissance envers une ville qui, leur ayant attribué une bourse, a contribué à leur promotion sociale. Il se traduit par des retours fréquents, des prises de responsabilités dans la ville, par l'activation à Paris des liens artistiques et affectifs. Dans ce mouvement d'affirmation face au pouvoir central où toutes les catégories d'acteurs trouvent leur compte, l'art comme expression d'identité méridionale est un moyen de pression politique. Toulouse obtient de choisir les artistes auxquels la décoration de l'hôtel de ville sera confiée et cette victoire assied de façon durable la préférence qui sera donnée aux artistes locaux au nom d'une identité artistique spécifique. Mais, de façon discrète, elle installe la région dans une autosatisfaction qui finalement la tiendra à l'écart de tous les débats en matière d'arts plastiques ${ }^{4}$. Lorsque survient la Première Guerre mondiale, les grands artistes nationaux de Toulouse sont morts et les discours glorifient la tradition dont les maîtres locaux sont les garants. L'entre-deux-guerres n'enregistre aucun changement dans les options à la fois locales et identitaires prises par la région toulousaine en matière d'arts plastiques. Le monde de l'art local fonctionne en vase clos et les deux galeries qui voient le jour dans les années 1920 ne changent pas la donne 5 . En dépit d'un réel essor d'un point de vue économique et universitaire, la capitale est empêtrée dans une identité méridionale qui lui sert d'alibi. Avec la Seconde Guerre mondiale, les déplacements de personnes et d'œuvres ${ }^{6}$ constituent un appel d'air rendant cet enfermement satisfait plus difficile à tenir. Pourtant, dans la perception des témoins de l'époque, la véritable remise en marche de la vie artistique régionale vient de la presse nationale qui irrigue à nouveau les régions.

2. La pression que la Ville exerce pour imposer ses choix s'exprime particulièrement lors du voyage du président Carnot en 1891, dont le programme comprend la visite de l'École des Beaux-Arts de Toulouse, sous la houlette de J. Jaurès; présents à cette occasion, autour des hommes politiques et des acteurs économiques, les artistes issus des Beaux-Arts de Toulouse, dont L. Falguière, J.-P. Laurens, H. Martin pour ne citer que les plus éminents

3. Il s'agit de peintres originaires de la région et ayant atteint un renom national: J.P. Maurens, H. Martin, B. Constant, Debat-Ponsan, Falguière, Marqueste...

4. Durant ces années, L. Rivet montre que les seuls artistes dont les Toulousains voient les œuvres sont originaires de la Ville rose et appartiennent à la tendance académique.

5. Les deux galeries privées exposent pour l'essentiel les artistes de la Société des Méridionaux.

6. Des artistes connus transitent dans la région (notamment Matisse, Villon, Bellmer ou Zack et Zadkine) et les œuvres des musées nationaux mises à l'abri en province permettent aux Toulousains de découvrir une partie du fonds du Musée national d'art moderne. 


\section{Deuxième période: l'occitanisme ou la quête de modernité}

Après la Libération, un bouillonnement se fait sentir dans le milieu des créateurs. À Toulouse, Castres et Montauban, des collectifs d'artistes se créent et vont ouvrir les débats, signe qu'une nouvelle génération cherche à se faire entendre. L'abstraction est au cœur des préoccupations ainsi que des prises de position esthétiques ${ }^{7}$. Cette intense activité intellectuelle va se traduire à partir de 1950 par une série de manifestations et de rendez-vous ${ }^{8}$ qui signent le renouveau de la vie artistique locale. Si, dans son ensemble, le monde de l'art régional reste dominé par les traditionalistes, la dynamique se poursuivra dans la décennie suivante, notamment grâce à des figures qui animeront la vie artistique jusque dans les années $1970^{\circ}$. Au plan national s'amorce un virage déterminant avec, en 1959, la création du premier ministère des Affaires culturelles confié à André Malraux. Sur fond d'une attention nouvelle portée par l'État à la culture et aux arts plastiques ${ }^{10}$, les années 1950 à 1970 sont pour la région Midi-Pyrénées celles de l'élargissement de la vie artistique locale à d'autres villes et régions du Sud.

Cette ouverture et cette quête de la modernité ne sont pas séparables d'une nouvelle déclinaison de la thématique identitaire dont l'apogée aura lieu dans les années 1970; entre l'engagement pour la modernité artistique et les prises de position en faveur d'une identité occitane, les passerelles sont nombreuses. Ainsi, et pour ne citer que les exemples les plus marquants, F. Castan et C.P. Bru, deux acteurs essentiels du nouveau souffle artistique, sont également engagés dans le mouvement occitan ${ }^{11}$. Avec eux, les groupes d'artistes vont rencontrer les tenants d'un régionalisme qui refuse l'enfermement. Prenant une distance avec la méridionalité du début du siècle, l'un comme l'autre défendent l'idée que la revendication occitane ne doit plus signifier un refus des esthétiques contemporaines, mais doit au contraire penser la préservation de l'identité dans l'ouverture et l'échange.

À l'inverse de ce qui s'est passé au début du siècle, la défense de l'identité occitane dans la version des années 1950 à 1970 est militante, comme l'illustre le slogan omniprésent Volem viure al païs ${ }^{12}$. Ses acteurs ne sont plus des édiles politiques ou artistiques ayant à cœur de conserver leurs positions, mais des «nouveaux occitanistes» issus de la

7. Comme en attestent les écrits et manifestes de l'époque, particulièrement ceux de C.P. Bru, considéré comme le théoricien de l'École castraise d'art abstrait.

8. C'est le cas des trois salons créés à cette période: le Salon Art nouveau; le Salon Art présent et le Salon Rencontres d'art.

9. C.P. Bru (philosophe et peintre), P. Arribaut (critique d'art à La dépêche), H. Lhong (amateur d'art et animateur d'espaces d'exposition), J. Fauché et V. Duguy (peintres); figures auxquelles il faut ajouter F. Castan (écrivain et ardent promoteur de l'occitanisme) et Marcelle Dulau (peintre).

10. La mise en place du $1 \%$ répond au souci d'une intégration de la production artistique dans les programmes de bâtiments, et les maisons de la culture annoncent également le souci de conduire le local vers une plus grande maîtrise de la diffusion en matière culturelle.

11. Entre 1950 et 1960, le premier est rédacteur en chef de la Revue d'Oc, le second est rédacteur des Annales de l'Institut d'études occitanes.

12. Ce slogan qui signifie "nous voulons vivre au pays» est également le nom donné au mouvement socialiste occitan qui naît en 1974 à Montpellier. À ce sujet, nous renvoyons à l'ouvrage de Pierre Lagarde (Lagarde, 1977). 
Libération dont beaucoup sont liés au Parti communiste français ${ }^{13}$. Pour eux, le problème occitan n'est pas seulement culturel, il est également socioéconomique et administratif, et il faut forcer l'autorité de l'État à s'engager dans la voie d'une décolonisation.

Aux côtés de ces militants de la cause régionale, les artistes qui se battent pour une expression contemporaine sont plus que jamais engagés dans la contestation politique. Les Écoles d'art, comme les autres établissements d'enseignement supérieur partout en France, sont le lieu de confrontations très violentes. Le rejet des institutions, accusées d'immobilisme, s'accompagne de celui de la centralité, favorise les revendications identitaires et le souci de démocratisation de l'art. C'est dans cette veine qu'il faut comprendre l'apparition de lieux nouveaux et de nouvelles manifestations dans toutes les régions, et particulièrement dans le Grand Sud. C'est aussi à la perte de vitesse du marché de l'art français qu'il faut rattacher les remises en cause de la suprématie parisienne qui se font jour dans la presse nationale spécialisée. En 1972 et 1974, deux dossiers sont consacrés à la vie artistique des provinces: le magazine animé par Jean Clair, Chronique de l'art vivant, fait paraître un numéro spécial intitulé «La province bouge» qui salue la vitalité des regroupements d'artistes de province qui entendent «créer un réseau parallèle pour faciliter des échanges impossibles avec les organes locaux passéistes». J. Lepage, militant actif et précoce de cette prise de conscience ${ }^{14}$, lui emboîte le pas dans le magazine Opus: dénonçant la «centralité extrême» des crédits muséaux, il soutient la légitimité des revendications souvent radicales des artistes provinciaux.

En Midi-Pyrénées, un monde de l'art contemporain commence à apparaître à travers des rendez-vous réguliers, des manifestations ponctuelles ou des structures d'exposition ${ }^{15}$, mais l'initiative ne vient pas des collectivités locales qui continuent à privilégier un art très traditionnel. Le rendez-vous le plus important pendant une décennie est $\mathrm{La}$ Mostra del Larzac. Il naît en 1970 de l'énergie de F. Castan et M. Dulau' ${ }^{16}$. Située sur le plateau du Larzac en Aveyron qui deviendra un haut lieu de la contestation régionaliste ${ }^{17}$, La Mostra del Larzac a pour ambition d'affirmer la spécificité d'une création occitane en favorisant des «relations horizontales», et de promouvoir le rayonnement régional à l'échelle internationale. La Mostra, relayée par des critiques parisiens qui, dans cette ambiance générale de découverte des «provinces», n’hésitent plus désormais à arpenter les régions, trouve rapidement le soutien du ministère de la Culture.

Les années 1970 marquent l'apogée du mouvement régionaliste, en même temps que les véritables prémices de la contemporanéité en région Midi-Pyrénées. C’est sur cette génération d'acteurs que la décentralisation prend appui à partir des années 1980

13. C'est notamment le cas de F. Castan.

14. En 1962, J. Lepage contribue à créer le Festival des arts plastiques à Antibes.

15. Ainsi, parmi les lieux d'exposition qui se créent, deux sont orientés vers la diffusion de l'art international — l'Abbaye de Beaulieu dans le nord du Tarn-et-Garonne et le Parvis à Tarbes — et un vers la promotion de la création régionale-l'enAc.

16. Animateurs du Salon Art nouveau de Montauban dans les années 1950-1960.

17. Le choix de ce relais de poste ne doit rien initialement aux évènements qui ont rendu le plateau du Larzac célèbre. Mais lorsque, entre 1972 et 1974, les luttes contre l'armée culminent, l'afflux de visiteurs viendra asseoir une situation géographique qui au départ pouvait être considérée comme un handicap. 
pour imposer définitivement un monde de l'art contemporain en région. La décentralisation des années 1980 mise en place par le nouveau gouvernement socialiste est la réponse à un problème devenu évident avec la prise de parole des provinces ${ }^{18}$.

\section{Troisième période: la décentralisation ou l'identité escamotée}

Avant même qu'elle ne soit effective, la décentralisation a provoqué dans les milieux artistiques régionaux un bouillonnement qui conforte le dynamisme du circuit associatif. Les artistes présents à la fin des années 1970 participent activement à la réorganisation du paysage artistique régional, soutenus en cela par des critiques, des écrivains, des passionnés. Les jeunes représentants d'institutions, suivant la voie dessinée par C. Mollard à la Direction des arts plastiques, sont très attentifs au terreau local dont certains sont issus. La politique de soutien aux initiatives locales, définie par la DAP, porte ses fruits. Dans le conflit souvent, mais aussi dans l'enthousiasme, les actions s'organisent et la recherche de collaborations et d'échanges sur un axe sud allant de Barcelone à Milan fait écho à la revendication régionaliste. L'apparition d'une critique forte est une autre nouveauté ${ }^{19}$. Au cours de cette même décennie, le dynamisme est général: le circuit marchand connaît un développement sans précédent qui bénéficie à toutes les expressions artistiques, des plus traditionnelles aux plus contemporaines; portées par l'engouement général en faveur des arts visuels, les municipalités ouvrent des espaces d'exposition et les vocations artistiques n'ont jamais été aussi nombreuses.

Les années 1980 font apparaître deux modifications essentielles imputables à la décentralisation ${ }^{20}:$ l'instauration d'un monde de l'art contemporain régional qui détient désormais le pouvoir de qualification des artistes et des œuvres; l'apparition d'une nouvelle catégorie d'acteurs dans la vie artistique. D'abord individuels ou associatifs dans les débuts de la décentralisation, les acteurs qui s'imposent ensuite sont les représentants des institutions décentralisées que sont le FRAC (Fonds régional d'art contemporain), la DRAC (Direction régionale des affaires culturelles), et les CRAC (Centres régionaux d'art contemporain). Encouragées par la DRAC, les collaborations entre les différents participants du monde de l'art contemporain se stabilisent, permettant ainsi la mise en place d'un maillage institutionnel régional. Le rattrapage de la région en termes de structures et le rééquilibrage entre départements entraînent la croissance en effectif des médiateurs culturels. Cette croissance, associée à la prééminence qu'ils vont acquérir dans le paysage artistique local, ne signifie pas que l'on assiste à « un dessaisissement de l'État au profit des régions» puisque celui-ci maintient «un type d'influence traditionnel» et «l'irrigation en argent [est] inséparable de

18. Même si, comme le souligne R. Moulin (Moulin, 1992), le souci de conduire le plan local vers une plus grande maîtrise de la diffusion en matière culturelle était déjà perceptible avec la mise en place des maisons de la culture à partir des années 1960 puis des chartes culturelles en 1974.

19. Deux magazines, Pictura Edelweiss et Axe Sud, acquièrent un rayonnement qui dépasse la région, à la fois par une diffusion nationale et par les collaborations dont ils s'entourent. Rapidement, Axe Sud s'enrichit d'une galerie associative. Le couplage galerie-revue permet un relais efficace vers l'extérieur et parmi les artistes locaux qui ont bénéficié de son soutien dans leurs débuts, certains confirmeront leur parcours par la suite.

20. Nous renvoyons pour cela au chapitre 2, deuxième partie de Azam, 1998. 
l'irrigation en hommes» (Moulin, 1992, p. 97). Dans ce contexte, la question de l'identité culturelle régionale prend une tonalité différente. Les institutions décentralisées sont autant de points par lesquels passe une modernisation de la vie artistique en MidiPyrénées, mais elles sont également des lieux où se joue désormais, par le truchement des fonctionnaires de l'art, la question de l'identité régionale face à Paris. C'est particulièrement le FRAC qui, dès le début, cristallise cette problématique.

Mis en place dans le début des années 1980, les FraC sont avant tout des fonds d'investissements en œuvres d'art destinés à assurer la relance du marché et leur mission principale est l'acquisition d'œuvres d'art contemporain. Placés sous le signe de la décentralisation et de la démocratisation, ces achats sont désormais assurés par des comités d'acquisition dont la composition multiplie les personnes prenant part aux décisions ${ }^{21}$. La première période du FRAC (1983/1985) est celle d'une ouverture de la région sur l'extérieur. Pour désenclaver la région et la rendre présente sur l'échiquier national et international, commence une stratégie d'échanges ${ }^{22}$ de l'Italie à l'Espagne, en passant par le sud de la France. Cette idée reprend le mouvement amorcé dans les années 1970, notamment avec La Mostra. Dans la logique des orientations décidées par C. Mollard, les personnalités locales actives dans la décennie 1970 sont impliquées dans les institutions naissantes: sur les dix membres qui composent les premiers comités d'acquisition figurent quatre personnalités locales reconnues pour leur action en faveur de l'art contemporain ${ }^{23}$.

Début 1985, des divergences apparaissent quant à la mission du FRAC. Le conseiller artistique régional souhaite privilégier l'acquisition d'œuvres d'artistes vivants incontestés pour sensibiliser des publics peu au fait de la création contemporaine. Cette position heurte les militants de la décentralisation qui estiment réduites à la portion congrue les sommes consacrées aux acquisitions de la création plastique régionale. Ces divergences de vue sur les règles d'acquisition du FRAC vont entraîner en 1985 la démission des personnalités locales. Plus largement, on assiste à un rétrécissement dans la composition successive des comités d'acquisition du FRAC: numérique d'abord ${ }^{24}$, géographique ensuite ${ }^{25}$. Il s'agit également d'un rétrécissement dans la diversité du type d'acteurs.

21. Avant la régionalisation, une quinzaine de personnes décidaient des achats publics en matière d'art contemporain; avec la régionalisation, c'est, en vingt-deux lieux différents, environ deux cent cinquante personnes qui participent à la décision (Moulin, 1992, p. 136 à 144).

22. Qui se concrétise par l'invitation de personnalités étrangères dans le comité d'acquisition, par des expositions communes avec les FRAC Languedoc-Roussillon et Aquitaine, par l'attribution de bourses pour des séjours d'artistes en Espagne et en Italie notamment, par des subventions pour la participation à des foires internationales dans ces mêmes pays.

23. M. Battle - directeur de publication d'Axe Sud, G. Bonnefoi-directrice de l'Abbaye de Beaulieu, F. Castan — fondateur de La Mostra Del Larzac, P. Manuel_critique d'art pour Pictura Edelweiss et Axe Sud, entre autres.

24. Composé de douze membres initialement, le comité en comptera finalement cinq en 1990: le directeur du FRAC, le conseiller artistique régional, et trois représentants de lieux institutionnels en Midi-Pyrénées.

25. Le comité d'acquisition comportait dans les premières années deux personnalités étrangères — une personnalité espagnole et une personnalité italienne. De 1987 à 1989, les deux invités extérieurs viennent de régions voisines-Aquitaine et Languedoc-Roussillon. À partir de 1990, le comité ne comporte plus de personnalités invitées. 
Dans ce mouvement qui voit les représentants d'institutions prendre le contrôle du comité d'acquisitions, il y a plusieurs explications au premier rang desquelles figure la difficulté à trouver un accord dans un comité pléthorique où cohabitent des sensibilités esthétiques différentes. S'ajoutent à cela des éléments qui ont trait à la logique propre à chaque catégorie d'acteurs. D'un côté se trouvent les acteurs locaux qui se sont battus contre la centralité esthétique et en faveur de la création contemporaine régionale. Leur participation au comité d'acquisition du FRAC signe une reconnaissance de leur action, mais l'indifférence qu'ils ont eu à affronter est si profonde que la tentation est toujours présente chez eux de revenir à une vision géographiquement plus étroite de la création $^{26}$. De l'autre côté se trouvent les représentants d'institutions dont la carrière est étroitement liée à la visibilité des actions et à l'alignement sur des critères de choix reconnus à l'échelle nationale, voire internationale. Chez eux, la tentation est inverse d'effectuer des choix d'artistes confirmés au niveau national et international. Leur logique rejoint celle des élus qui trouvent avantage à s'abriter derrière les options défendues par ces spécialistes peu suspects de provincialisme. Pour ces deux catégories d'acteurs, les achats prestigieux participent au rayonnement personnel autant qu'au rayonnement de l'institution qu'ils représentent. Une autre raison fait des représentants d'institutions culturelles et des élus des alliés objectifs: comptables de la saine utilisation de fonds publics, ils tendent à orienter les acquisitions du FRAC vers la constitution d'un fonds patrimonial. L'évolution dans ce sens que connaît l'ensemble des FRAC amène d'ailleurs C. Mollard ${ }^{27}$ à rappeler en 1986 aux responsables de ces institutions que l'enrichissement du patrimoine n'est pas la finalité. Ce virage patrimonial sera finalement pris avec l'arrivée du nouveau délégué aux arts plastiques au ministère de la Culture ${ }^{28}$. L'exemple du FRAC illustre la façon dont le crédo identitaire perd progressivement du terrain, ce qui, au demeurant, semble être dans l'ordre des choses pour la majorité des participants. Pour eux, comme pour les jeunes artistes des années 1980 qui se reconnaissent de moins en moins dans le combat de leurs aînés, la question s'est déplacée: ce qui doit être défendu n'est pas tant une identité artistique régionale, au demeurant introuvable, que la possibilité matérielle de produire un art contemporain en région.

Des deux cotés donc, la création contemporaine régionale se trouve gênée. Elle se heurte d'abord aux médiateurs de l'art qui privilégient des actions visibles et d'envergure clairement nationale, voire internationale ${ }^{29}$. Elle se heurte ensuite aux édiles locaux,

26. Ainsi qu'en témoigne cette phrase d'un membre de la commission d'acquisition, dénonçant «les acquisitions faites à certains plaisantins, tels que Ben, alors que l'on refuse d'acquérir une sculpture d'une véritable artiste comme Odile Mir».

27. Sources: Actes des troisièmes rencontres nationales des FRAC, Lyon, 10 et 12 mai 1986.

28. Il s'agit de Dominique Bozo qui succède à Claude Mollard en 1986.

29. C'est par exemple le cas de la programmation du Centre régional d'art contemporain qui fut implanté sur la commune de Labège; c'est également le cas de la programmation de l'actuel Musée d'art moderne et contemporain de Toulouse, appelé Musée des Abattoirs. La logique des galeries privées défendant l'art contemporain n'est pas très éloignée de celle des institutions locales: leurs contraintes financières les amènent à présenter des artistes contemporains dont la réputation est assise, au moins au niveau national. 
peu formés à des expressions novatrices et peu enclins à soutenir un art contemporain qui dérange et que l'on accuse — et non sans raison — d'élitisme. Ce constat est peu réjouissant: la décentralisation a donné sa légitimité à un monde de l'art contemporain dans la région, mais le dynamisme qu'elle avait un temps soutenu s'est essoufflé et le salut des artistes qui refusent le traditionalisme passe toujours par le départ de la région. Par ailleurs, la décentralisation, en étant finalement l'aboutissement de la montée en puissance des régions, se présente comme une réponse aux revendications régionalistes qu'elle désamorce ${ }^{30}$.

\section{LES COM POSANTES DU CRÉDO IDENTITAIRE}

\section{Édiles et artistes : qui mène la danse?}

Durant la première partie du siècle, les artistes viennent en renfort d'un simple jeu d'influence conduit par les édiles locaux pour imposer des choix face à la centralité parisienne. Durant la période qui s'ouvre au début des années 1950 et culmine durant les années 1970, les artistes mènent aux côtés des occitanistes le mouvement de contestation contre Paris, et plus largement contre une tradition politique, esthétique et organisationnelle asphyxiante. C'est dans cette optique que les acteurs du mouvement occitan et les partisans de la contemporanéité esthétique orientent les échanges préférentiellement en direction du sud de la France. Durant ces périodes successives, les artistes savent œuvrer collectivement pour peser sur une configuration donnée du jeu local. Qu'il s'agisse d'être présents pour l'embellissement de la ville comme au début du siècle, ou qu'il s'agisse d'animer la vie artistique par les manifestations ou les associations qu'ils créent en 1950, à la fin des années 1970, ainsi qu'au début des années 1980, ils adoptent des stratégies collectives, seules à même de rendre possible une quelconque action. Reste que ces stratégies durent peu, que leur rayonnement est essentiellement local, et les artistes n’en sont pas toujours bénéficiaires.

Ainsi, au début du siècle, comme au début des années 1980, les artistes tirent parti de leur appartenance régionale puisqu'ils bénéficient, soit de commandes, soit d'un dynamisme exceptionnel qui offre à la jeune génération des opportunités multiples ${ }^{31}$. Mais entre les années 1950 et 1970, l'indifférence des pouvoirs publics vient à bout de trois générations d'artistes parmi ceux engagés régionalement pour redonner un souffle à la vie artistique ${ }^{32}$. En effet, à chaque décennie pendant cette période, de jeunes artistes participent à la dynamisation de la vie artistique locale, mais l'absence d'écho décourage les énergies. Bien sûr, les artistes connaissent individuellement des fortunes

30. Un signe de cet affaiblissement se trouve notamment dans l'indifférence, voire le rejet du discours identitaire, par la génération d'artistes qui entament leur parcours dans le début des années 1980: pris dans un tourbillon sans précédent, ils ne se reconnaissent pas dans le combat de leurs aînés.

31. Il faut souligner que ces jeunes artistes récoltent, en partie, les fruits de l'engagement des artistes de la génération précédente dans l'organisation du circuit associatif.

32. À l'image de Marfaing, par exemple, on peut remarquer que n'en réchapperont finalement que les individualités qui choisissent de partir. 
et des parcours divers, mais l'existence d'un effet générationnel fort sur la visibilité des artistes et leur possibilité de travailler dans cette région montre à quel point leurs actions et leur mobilisation ont besoin pour se pérenniser d'obtenir un soutien politique, à quelque niveau que ce soit.

De ce point de vue, le manque actuel d'artistes reconnus nationalement ou internationalement — que l'on peut identifier à la région ou qui revendiquent ces liens montre a contrario l'importance d'un soutien politique local. Là encore, la comparaison avec la première partie du siècle s'impose: alors que la capitale régionale entretient des liens forts avec «ses» artistes partis à Paris, durant la deuxième moitié du siècle, elle ne cherche pas à faire participer les artistes à la vie artistique locale. Dans le même ordre d'idées, les artistes inscrits dans de nouvelles formes d'expressions ne voient pas leurs œuvres achetées par le musée, et même ceux dont la reconnaissance est établie ailleurs ne trouvent pas forcément un écho sur leur terre d'origine ${ }^{33}$. Dans ces conditions, rien d'étonnant à ce que ces artistes ${ }^{34}$ ne manifestent pas d'attaches particulières avec la région, encore moins un sentiment d'appartenance à une quelconque communauté artistique ou culturelle.

Dans ce pas de deux entre arts plastiques et région toulousaine, les artistes débutent souvent la danse, mais ils la mènent rarement de façon durable. Son rythme, rapide ou lent, échappe aux artistes: il est imposé par les édiles avant qu'une nouvelle catégorie d'acteurs - les médiateurs culturels — ne supplante celle des artistes face aux politiques locaux. De fait, toutes les périodes où l'on observe un relatif dynamisme de la vie artistique locale s'inscrivent dans un contexte qui dépasse largement les frontières régionales. Dans un domaine différent, ce constat rejoint celui de M. Grossetti: analysant les conditions de mise en place du système scientifique local, il montre que «les évolutions du système d'action se coulent pour l'essentiel dans les phases de stabilité ou de rupture du système national» (Grossetti, 1994, p. 213).

\section{Les avatars du discours identitaire}

Parmi les différents facteurs qui expliquent les raisons pour lesquelles les stratégies collectives ont été si peu fructueuses pour les artistes, se trouve certainement la difficulté à donner une forme proprement plastique à l'idée d'identité culturelle et ce n'est pas un hasard si, à l'image du Félibrige, le fer de lance des combats pour l'identité culturelle est la littérature. Dans le domaine des arts plastiques, l'existence d'un courant artistique est une des formes que l'identité culturelle peut revêtir pour se rendre visible et l'histoire de Midi-Pyrénées est émaillée de ces tentatives dont le succès est faible.

Nous avons vu apparaître au début du siècle une «école de Toulouse». Dans leur action pour faire fléchir le pouvoir central et afin de légitimer leurs choix artistiques,

33. Ainsi, la région et particulièrement la capitale régionale ont mis du temps à les reconnaître comme faisant partie du «patrimoine» local: en effet, si des œuvres de ces artistes_-Marfaing, Bissière, Soulages, par exemple — ont bien été acquises par les Augustins, ces acquisitions sont toujours très tardives.

34. Ou d'autres encore auxquels on pourrait ajouter Bustamante pour la jeune génération. 
les acteurs - hommes politiques mais aussi des artistes — se sont évertués à trouver les signes d'une filiation plastique entre cette période de rayonnement et la charnière du $\mathrm{Xx}^{\mathrm{e}}$ siècle, se référant au $\mathrm{XVII}^{\mathrm{e}}$ siècle qui connut l'apogée de la peinture toulousaine. Cette idée, si elle contribue à imposer les choix toulousains contre Paris, connaîtra une fortune purement locale.

Après la Libération, c'est à deux tentatives concurrentes pour asseoir et structurer l'éveil régional que l'on assiste. À la fin des années 1950, C.P. Bru, le premier, évoque l'idée d'une «école castraise d'art abstrait». De son côté, H. Lhong parle en 1964 d'une «école de Toulouse», née dans les années 1950, et dont la manifestation «Présence 1» donne l'impulsion ${ }^{35}$. Aucune de ces deux tentatives ne parviendra à s'imposer.

Au cours des deux dernières décennies, l'occitaniste F. Castan ${ }^{36}$ et le peintre, médiateur culturel et critique B. Meyer Himhoff renouvellent l'entreprise, en l'élargissant chacun de façon différente. F. Castan regroupe sous le terme hyperabstraction «une cinquantaine de peintres et de sculpteurs appartenant généralement à la région » et propose une possible solution «au problème laissé en suspens par l'abstraction des années 50 ». Dans son essai de genèse des œuvres, il inclut des artistes reconnus de la région: Bissière, Soulages et Marfaing. De son côté, B. Meyer Himhoff cherche à établir un lien entre les principaux acteurs du renouvellement de la vie artistique depuis la Libération $^{37}$. Dans sa volonté d'assembler les différentes pièces de ce patchwork identitaire, il écrit:

Il ne s'agit nullement de dégager les éléments d'une école régionale puisque, dans leur cohérence, les pratiques et les théories picturales dépassent les limites de la région et sont confrontées à un mouvement d'idées générales à l'échelle du monde artistique contemporain, mais peut-être d'entreprendre une enquête sur les sources de l'art vivant dans une région qui depuis quelques mois se transforme avantageusement.

Qu'elles soient baptisées «écoles» ou non, ces diverses tentatives seront reprises de façon sporadique au niveau régional et connaîtront un succès très limité. Pourtant, le discours d'une identité particulière subsiste avec des déclinaisons diverses. Dans sa version contemporaine, l'identité est désormais délayée dans l'existence d'un grand Sud dont le dynamisme artistique est devenu visible, idée qui est reprise par les institutions elles-

35. Dans l'introduction du catalogue de l'exposition, Peinture à Toulouse en 1964, il s'emploie dans un article à démontrer l'existence d'une école toulousaine de 1950. S’efforçant de rendre visibles des «liens de parenté», l'auteur s'emploie à montrer la contemporanéité de ces jeunes artistes: «Par une sorte de synchronisme troublant beaucoup plus que par influence, ils participaient d'un expressionnisme misérabiliste qui s'imposait à Paris avec le lancement de Bernard Buffet, lequel venait de peindre ses toiles majeures. Ce synchronisme prouve bien que nous avancions à l'heure, à savoir que ces peintres se voulaient bien présents dans les problèmes de la peinture de l'époque.»

36. Source: F. Castan, La Mostra $\mathrm{n}^{\circ} 22,1987$ et n 33/34, 1990.

37. B. Meyer Himhoff, dans un article, écrit à propos de C.P. Bru «... peintre mais aussi théoricien qui a publié plusieurs ouvrages ... l'apport de C.P. Bru est, ici, largement reconnu. Il fut soit l'initiateur, soit le participant actif de manifestations, groupes ou associations depuis les années 1950 dans la région toulousaine, en compagnie du peintre J. Fauché, de H. Lhong, et à partir des années 1960, du peintre M. Dulau et de F. Castan; plus récemment (1975), C.P. Bru fut membre actif de Peinture itération avec G. Boyer, C. de Cambiaire et R. Métivet» (Himhoff, 1983, p. 103). 
mêmes. Avec l'ouverture que la décentralisation amplifie, le combat régionaliste perd sa cible favorite: l'État centralisateur. Le problème de l'identité s'atténue et se déplace ${ }^{38}$.

D’une façon générale, passé l'enthousiasme des premières années de la décentralisation, les discours font état de la situation défavorable dont la région n'arrive pas à s'extraire. Dans le même temps, il n'échappe pas aux acteurs du monde de l'art régional que le crédo identitaire éprouve des difficultés lorsqu'on cherche à le conjuguer avec contemporanéité. L'entretien accordé par M. Bellit ${ }^{39}$ à Axe Sud ${ }^{40}$ en donne l'illustration, constatant la nécessité pour l'identité culturelle de se battre, d'une part, sur le front intérieur contre la tentation toujours présente d'enfermement ou de «pseudoréactivation du passé», et, d'autre part, sur le front extérieur puisqu'il faut désormais travailler à positionner la région sur la scène internationale.

Les avatars que connaît ce discours durant le $\mathrm{xx}^{\mathrm{e}}$ siècle révèlent d'une part la ressource qu'il peut représenter pour les artistes à certaines périodes de l'histoire, mais également les dangers et les limites d'une telle construction. Alors que pendant la première partie du siècle, le discours identitaire justifie la fermeture et l'isolement de la région sur elle-même, dans les années 1950 jusqu'à 1970, les activistes occitans et les plus en vue des artistes plasticiens vivant en région œuvrent avec des intensités variables au nom de l'ouverture et du brassage ${ }^{41}$. Mais dans ce mouvement, à mesure que les énergies des artistes et des amateurs s'épuisent à essayer de promouvoir un art vivant et en prise avec les problématiques de l'époque, le crédo d'une spécificité culturelle se délaye au point finalement de se perdre dans un Sud trop grand. Cet affaiblissement est particulièrement clair pour les artistes en arts plastiques: il leur est moins évident de revendiquer une expression esthétique spécifique, contrairement à d'autres formes d'expressions, telles que la littérature ou la musique, qui s'appuient directement sur la langue. En changeant de domaine artistique, le discours occitan perd une partie de son assise; en changeant de registre - de la protection d'une spécificité à l'ouverture vers le contemporain - le discours identitaire se fait plus ténu. C'est à ce moment-là d'ailleurs que s'opère clairement le passage de la question identitaire à la question du retard culturel. En quelques décennies donc, on assiste à un renversement paradoxal:

38. C’est ainsi que P. Manuel souligne le déséquilibre des relations de Midi-Pyrénées avec le reste de l'axe du Grand Sud: «Derrière des choix esthétiques commandés par une forme d'homogénéité et une relation à l'actualité européenne, l'enjeu véritable est l'affrontement politique et économique de la région avec, d'une part la Catalogne - qui est quasiment un État et assure une politique culturelle nationale — et d'autre part Milan, une des villes dont le dynamisme économique et idéologique, par ses galeries et ses revues, est le plus fort en Europe. Ce type d'échange, les économistes du tiers-monde l'appellent échange inégal» (Manuel, 1984, p. 84).

39. Directeur d'un lieu institutionnel depuis 9 ans, il venait d'être nommé chargé de mission aux affaires culturelles de Midi-Pyrénées.

40. Source Axe Sud, n 4/5, 1982.

41. Pour être complet, il faut préciser que cette tentation identitaire n'est pas seulement le fait des artistes ou personnes engagées dans le segment avancé de l'art régional. Elle est aussi le fait d'artistes ou de personnes qui, défendant une expression très traditionnelle, voient dans ce type de production l'affirmation d'une résistance à un art contemporain. Souvent, et avec plus ou moins de véhémence, le refus des «gens du ministère de la Culture» ne s'apparente pas au refus de la centralité comme étouffoir de la créativité régionale; pour ces acteurs, il ne s'agit pas d'une opposition à Paris mais d'une opposition à un art qui «dégénère». 
alors que les arguments identitaires avaient servi de ferment à une vie artistique redynamisée, ils vont être progressivement renvoyés au statut d'oripeaux tout juste bons à habiller la thèse du retard culturel.

\section{Le «retard» comme identité régionale?}

La chronologie a fait apparaître entre les deux grandes périodes de la vie artistique en Midi-Pyrénées un déplacement du pôle de certification de la valeur artistique: celuici a glissé de la traditionnalité, dont la prééminence décline à partir des années 1960, à la contemporanéité qui s’impose dans les années 1980. Entre ces deux périodes, une idée revient de façon insistante dans les discours des acteurs: celle d'un «retard». Ce terme renvoie alternativement à un retard dans le dynamisme créatif ou à un déficit dans la mise en valeur de ce dynamisme créatif, cela dépend du point de vue adopté. Ce retard est-il une spécificité de Midi-Pyrénées? Avec quels arguments les acteurs et analystes de la vie artistique alimentent-ils cette thèse ou la réfutent-ils?

Pour la première partie du siècle, si l'on se range à l'avis de L. Rivet, il existe un retard qui persiste au moins jusqu'en $1939^{42}$. Pour la seconde moitié du siècle, les éléments dont nous disposons appellent une lecture moins nette. Les témoignages que nous avons recueillis insistent tous sur le dynamisme que connaît la région après la Libération et sur le fait que les recherches engagées par les peintres sont en prise avec les problématiques artistiques du moment. On peut, bien sûr, s'interroger lorsque l'on sait que ce qui occupe les esprits régionaux à cette époque est le débat abstraction/figuration et voir dans ces peintres abstraits régionaux des années 1950 des «suiveurs », ainsi que les qualifie R. Moulin ${ }^{43}$. Pourtant, si l'on suit G. Monnier (Monnier, 1986), la réalité est plus nuancée et il n'est pas sûr qu'ailleurs la situation soit très différente. Dans l'aprèsguerre, les initiatives au niveau national orientées vers la modernité demeurent limitées et la reconnaissance des artistes modernes ${ }^{44}$, au premier rang desquels Picasso ${ }^{45}$, serait une sorte de «dette morale» envers des artistes qui ont manifesté une «opposition culturelle à l'occupant». Mais, selon l'auteur, la France n'est pas pour autant sortie de l'indifférence et de la pesanteur antérieures ${ }^{46}$ et la timidité de l'ouverture est, selon toute vraisemblance, commune à l'ensemble des provinces de France, tant il semble qu'à Paris même, celle-ci soit limitée.

42. L'auteur établit cette thèse en analysant, notamment et pour toute la première partie du siècle, la politique d'acquisitions des musées locaux, la politique des commandes publiques et la programmation des divers salons régionaux entre le début du $\mathrm{xx}^{\mathrm{e}}$ siècle et l'année 1939.

43. En effet, ainsi que le fait remarquer R. Moulin, le débat abstraction/figuration semble à Paris largement dépassé puisque « [...] dans l'esthétique de la rupture et de la priorité qui définit la modernité [...] les suiveurs de l'abstraction des années 50 n'ont pas résisté au double choc du retour en force de la figuration et de la crise boursière dans la première moitié des années 60" (Moulin, 1992, p. 338).

44. Avec l'entrée dans les collections nationales d'œuvres de H. Matisse, Bonnard, Braque, Rouault, J. Gris, F. Léger (Monnier, 1986).

45. Auquel une salle entière est consacrée lors du Salon d'automne de la Libération (Monnier, 1986, p. 245).

46. Au cours de cette même période, en province, une seule exposition est remarquable par l'audace des choix proposés: elle se déroule en Avignon en 1947. 
Comment la situation a-t-elle évolué à la fin du siècle? L'histoire des régions en matière d'arts plastiques reste à faire et il n'existe pas de travaux permettant de situer Midi-Pyrénées par rapport à d'autres régions de la France. Il est malgré tout possible de se faire une idée de la perception que les acteurs ont de la situation régionale à travers la presse spécialisée. Nous avons vu que le réveil provincial occasionne deux dossiers, signés par J. Lepage, Art Vivant en 1972 et Opus en 1974: l'auteur situe les prémices de la poussée des régions à la fin des années 1950 avec le Nouveau réalisme niçois, sa diffusion dans la Loire à partir du milieu des années 1960 et, s'appuyant sur la voie ouverte par Support-Surface, son extension le long de la Méditerranée en 1970. Entre 1969 et 1970 le mouvement a fait tache d'huile ${ }^{47}$. Cette analyse de la situation en régions indique que Toulouse reste en deçà du bouillonnement général et suit un mouvement déjà bien entamé partout; or, les témoignages de protagonistes que nous avons recueillis présentent pour leur part l'action toulousaine comme étant à la pointe de cette volonté de création d'échange sur un axe sud. La divergence d'appréciation du dynamisme régional nous inspire la réflexion suivante: ces numéros spéciaux consacrés à la vie artistique en région ne peuvent avoir été réalisés que par le biais de réseaux personnels qui se tissent et permettent à l'information de circuler. L'on peut en déduire que, si la vie artistique a été plus intense que ne le laissent supposer les informations dont nous disposons, la région a manqué de relais sur l'extérieur pendant la première vague du mouvement provincial, relais dont ont manifestement bénéficié Limoges et Tours, deux villes pourtant plus petites et ne disposant pas plus que Toulouse d'une situation géographique favorable.

Un autre élément, susceptible d'éclairer cette question, concerne la faiblesse des groupes artistiques pendant cette période. Contrairement à d'autres régions où les groupes semblent plus stabilisés, les artistes de la région donnent plutôt l'impression d'être en période de recherche d'axes qui pourraient être fédérateurs. Ce qui unit le seul groupe repéré à l'extérieur, le CAPT composé d'une vingtaine d'artistes, ne relève pas d'une communauté de sensibilité esthétique mais, ainsi que l'annonce le manifeste du groupe, d'une communauté de moyens que le groupe entend créer pour combattre «l'isolement de la région, l'absence de dialogue et l'indifférence des pouvoirs publics».

Dans les années 1990, la situation semble avoir peu évolué, si l'on se réfere à l'opération organisée par la revue Eighty en 1990, "Créateurs à suivre», qui présente une sélection d'artistes par régions. Le « retard», que certains acteurs voient comme une caractéristique permanente de Midi-Pyrénées, serait également celui d'autres régions. Dans ce numéro spécial, chaque jury régional, composé de deux membres, introduit la sélection d'artistes par un texte faisant le point sur les circuits de diffusion et plus largement sur la situation des arts plastiques de la région représentée. Il est évident que ces présentations n'engagent que leurs auteurs, mais il reste que chaque texte est une in-

47. Lyon, Nice et Tours organisent une succession de manifestations importantes qui investissent de préférence les espaces urbains. Viendront ensuite Montpellier, Limoges, Perpignan, Grenoble. Entre 1971 et 1972, s'ajoutent au mouvement Aix-en-Provence, Toulouse et St Etienne. 
dication du satisfecit d'acteurs par ailleurs engagés à divers titres dans la promotion de l'art contemporain et reconnus comme tels dans le cadre de cette opération.

Si l'on classe les textes en fonction du degré de satisfaction exprimé par les membres du jury, Midi-Pyrénées s'inscrit en queue de peloton ${ }^{48}$. Le jury de cette région ${ }^{49}$ met l'accent sur l'indifférence des politiques locaux qui mine le pari de créer dans cette région, «la grande affaire (des peintres de la région) est d'abord de survivre. Sans guère de lieux pour exposer, la mort les menace. L'école des Beaux-Arts est le foyer quasi unique de la jeune génération [...] un des problèmes majeurs est l'isolement [...] la quasi permanente frustration». Le jury de «Créateurs à suivre» insiste surtout sur le retard et l'environnement peu favorable comme lot commun à tous les artistes de MidiPyrénées depuis des générations et c'est dans cette résistance face à l'indifférence qu'il voit une filiation entre ces jeunes artistes des années 1980 et leurs aînés.

On pourrait multiplier les témoignages et les écrits qui reprennent de façon récurrente la question du retard comme caractéristique de la région et de sa capitale. Cette idée, sans toutefois faire l'unanimité, est assez largement partagée par les diverses catégories d'acteurs du monde de l'art. L'affrontement a véritablement lieu autour de la question de la nature de ce retard, certains arguant qu'il relève d'un manque de dynamisme créatif, d'autres d'une difficulté à rendre visible ce dynamisme créatif. Et s'agissant de cette dernière interprétation, les acteurs, et particulièrement les artistes, disposent en effet d'une multitude d'exemples concrets illustrant la profonde indifférence $^{50}$ de la région et de sa capitale envers les arts plastiques en général et les plasticiens régionaux contemporains en particulier. Ils attribuent également ce manque de visibilité à la domination dont ils estiment victimes les créateurs du secteur avancé en MidiPyrénées, rejoignant ainsi les analyses produites par un pan de la sociologie.

B. Poche, en analysant le milieu lyonnais (Poche, 1992), ne dit pas autre chose à propos d'une région pourtant reconnue pour son dynamisme: «Un art qui affiche désormais une origine visible est actuellement considéré comme un art de seconde zone... on se trouve alors devant le constat que les créateurs actuels sont condamnés au destin des postimpressionnistes lyonnais des années 1950, c'est-à-dire l'oubli. » ${ }^{51}$ Dans un contexte où les références plastiques sont celles d'un art international, donc délocalisé, la revendication d'une origine géographique et donc d'une identité culturelle, pour un artiste ou pour un groupe, est suspecte de provincialisme. À Lyon, dit encore B. Poche: «On n'expose pas les Lyonnais ou on expose sous ce titre ceux qui sont partis; ou alors on les expose bien, mais en faisant remarquer que ce sont des peintres lo-

48. Le classement fait apparaître dans les résolument "plus», par ordre alphabétique: Alsace, Auvergne, Bourgogne, Champagne-Ardennes, Limousin, Pays de Loire, Poitou-Charentes, Provence-Alpes-Côte d'Azur, Rhône-Alpes, Franche-Comté. Et dans les résolument «moins»: Bretagne, Centre, Corse, Midi-Pyrénées, Haute-Normandie, Picardie.

49. Il s'agit de J.M. Touratier, directeur du FRAC, et de H. Beulay, critique de La Dépêche.

50. Euphémisme en réalité, car c'est le plus souvent l'incompétence des édiles locaux et de leurs conseillers dont parlent les participants au monde de l'art. À titre d'exemple, on pourra lire le véhément article de Denis Millau, longtemps conservateur du Musée des Augustins de Toulouse (Millau, 1991).

51. Extrait de «La lettre de l'ACAL», janvier 90 (source Labatut, 1991, p. 68). 
caux. Aussi, on les isole! En les isolant, on les met en demeure de choisir entre s'en aller ou accepter de devenir un artiste régionaliste.» À ce titre, les artistes de MidiPyrénées connaissent les mêmes difficultés que ceux d'autres régions; de fait, nous avons vu que les revendications régionalistes sont particulièrement fortes dans les périodes d'affrontement entre une logique locale et une logique nationale et, dans ces moments, on assiste à la réactivation d'une identité culturelle. Ces crispations sur l'identité sont aussi le résultat d'une rébellion contre la difficulté d'échapper à cet enfermement auquel les contraint souvent une vie en province ${ }^{52}$.

Que l'analyse sociologique recoure au modèle de la domination ou que, dans une version plus récente, elle mobilise celui de la gravitation artistique (Menger, 1993), l'hégémonie parisienne sur la scène culturelle nationale n'a pas été affectée par la décentralisation des années 1980. Mais, au delà de la configuration historique qui oppose Paris aux régions, la thèse du « retard» est d'abord la traduction de la persistance d'une distorsion entre un centre et une périphérie. En attestent les débats actuels qui accompagnent la mise en place du processus de régionalisation ${ }^{53}$, et les problèmes évoqués par les acteurs d'ensembles organisationnels inférieurs en taille à la région (départements, communes, et nouveaux territoires organisés sous formes de "pays», "communautés d'agglomération »...) sont proches de ceux que l'on trouvait dans les débats des années 1970. Il est par exemple question du «retard» des départements par rapport à la métropole toulousaine, de la difficulté de reconnaissance des artistes vivants en zones rurales, de l'affirmation d'une identité spécifique pour des territoires plus restreints...

\section{CONCLUSION}

À l'issue de cette présentation des différents temps forts de l'histoire artistique locale et des éléments récurrents qui les lient entre eux, quels enseignements peut-on tirer des relations entre art et territoire en Midi-Pyrénées et, plus précisément, à quelles conditions le crédo identitaire peut-il représenter une ressource pour les artistes?

Dans le domaine des arts plastiques ${ }^{54}$, il apparaît clairement que ces relations sont déséquilibrées et, tendanciellement, en défaveur de l'art. Et s'il est arrivé que les arts plastiques servent de faire-valoir au territoire, il s'avère que l'inverse est moins vrai. Le déficit d'image dont souffre la région est réel ${ }^{55}$, ainsi qu'en atteste la thèse du retard culturel, que celle-ci soit fondée ou non. Le territoire de Midi-Pyrénées n'est pas pour les artistes plasticiens une ressource, au sens d'élément positif dont ils pourraient se

52. Ce que P. Bourdieu analyse bien: «Si la région n'existait pas comme espace stigmatisé, comme province, elle n'aurait pas à revendiquer son existence» (Bourdieu, 1980, p. 65).

53. Source: «Une journée culture en région. Jeudi 31 janvier 2002. Les actes», édité par le Conseil régional Midi-Pyrénées.

54. Il faut préciser que les domaines musicaux (classique et autres) et des arts de la scène (théâtre, danse et cirque) sont très dynamiques.

55. Ainsi, un envoyé du ministère de la Culture, avec lequel était abordée la condition des jeunes plasticiens en MP, n'a pas hésité à dire en aparté qu'il est préférable pour un jeune artiste de ne pas être identifié à la région. 
prévaloir collectivement ou individuellement et cela peut contribuer à expliquer pourquoi, depuis des générations, ils oscillent entre le départ de la région ou la revendication identitaire ${ }^{56}$.

Le parcours historique que nous avons proposé nous a permis de montrer que le discours identitaire peut être un ferment de l'action collective, mais tout aussi bien un alibi à l'immobilisme. Entre les différentes périodes que nous avons évoquées, nous avons pointé deux constantes de cette relation entre les arts plastiques et le territoire de Midi-Pyrénées: l'indifférence d'une région envers ses créateurs et la difficulté des acteurs du segment avancé de l'art local à établir un lien entre des productions censées posséder une coloration spécifique. Ces deux éléments se répondent mutuellement et jouent dans l'absence d'attention des médiateurs nationaux et internationaux envers une région cataloguée comme muette ${ }^{57}$. En effet, avec un tel déficit d'image dans le domaine des arts plastiques, comment les spécialistes de l'art contemporain pourraientils attendre de Midi-Pyrénées des artistes exceptionnels? Comment pourraient-ils susciter, par leurs encouragements ou leur attention, le développement de nouveaux talents? Cette pesanteur de l'histoire en matière de perception de la valeur artistique est observée à une tout autre échelle par A. Quemin ${ }^{58}$. Dans le monde de l'art contemporain international, il souligne que l'origine géographique des artistes — plus précisément leur nationalité - est intégrée par les experts comme un critère de valeur dans les évaluations esthétiques des œuvres (Quemin, 2001, p. 113), assurant ainsi l'omniprésence de certains états sur la scène internationale.

La thématique identitaire telle qu'elle est déclinée aux différents moments de l'histoire est une des formes que peut prendre l'action des artistes et des passionnés pour fédérer les énergies et provoquer le mouvement, mais l'insuccès de ces tentatives montre que la quête d'identité artistique reste à l'état d'ébauche si elle ne capte pas l'attention des autres acteurs locaux, au premier rang desquels se trouvent les élus.

Sur une scène extérieure, la thématique identitaire est également une réponse à une domination ressentie et dont le niveau d'imputation est variable. Ainsi, le crédo identitaire est mobilisé à différents niveaux, tantôt pour dénoncer la centralité parisienne, tantôt pour exprimer leur défiance envers un «axe sud» jugé trop englobant, tantôt encore contre une internationalisation ou une globalisation aliénante. Selon les scènes où s'élaborent les discours et où se situent les acteurs, l'identité défendue est une identité française, une identité spécifique au "grand Sud», ou encore au SudOuest, une identité occitane, à moins qu'il ne s'agisse d'une identité plus spécifiquement toulousaine... Dans cet exercice qui consiste à articuler un «ici» avec un «ailleurs» tantôt

56. Après un ralentissement dans les années 1980, la tendance au départ est redevenue d'actualité et parmi les jeunes artistes inscrits dans une expression contemporaine, beaucoup ont d'ores et déjà quitté la région.

57. Nous renvoyons à ce sujet à la lecture de Azam, 1998, première partie, chap. 3 .

58. «Le poids de l'histoire est donc primordial puisqu'un pays a d'autant plus de chances d'occuper une place enviable dans le palmarès des arts plastiques internationaux qu'il occupait auparavant une telle position» (Quemin, 2001, p. 84). 
national, tantôt international, la notion «locale» est à géométrie variable ${ }^{59}$. L'internationalisation, la mondialisation, et, en France, la régionalisation, donnent non seulement de nouveaux terrains de jeu aux participants du monde de l'art, mais également de nouveaux espaces aux arguments identitaires.

Ce crédo peut-il se décliner autrement que sur un mode défensif? Et compte tenu de leur logique respective, comment contemporanéité esthétique et particularismes locaux peuvent-ils s'articuler?

La contemporanéité est internationale, à l'image des académies informelles qui l'évaluent, et cette internationalité représente la principale caractéristique de l'art contemporain (Moulin, 1992 et Quemin, 2001) puisque «la validation par l'espace, par l'éloignement géographique, a désormais remplacé la validation par le temps qui caractérise l'art ancien» (Quemin, 2001, p. 14). L'extension spatiale suppose une circulation, non seulement des idées et des œuvres mais également des hommes, qu'ils soient artistes, médiateurs ou experts. Elle produit une tendance à l'homogénéisation des critères d'appréciation des œuvres, de sorte que les expressions artistiques contemporaines n'ont finalement que très peu à voir avec une implantation locale quelconque. Et, comme le souligne justement G. Bellavance dans son analyse du contexte québécois, toute l'ambiguité des artistes contemporains locaux est de «revendiquer simultanément l'internationalisme et le cosmopolitisme de leur régionalisme» (Bellavance, 2000, p. 18).

La vogue du multiculturalisme, la mise à l'honneur d'expressions artistiques considérées jusqu'à une période récente comme exotiques, pourraient faire croire à un effacement des frontières et à une prise en compte des productions artistiques indépendamment de leur origine géographique. Or l'arrivée des pays périphériques dans le concert international n'a sur le fond rien changé à la prééminence anglo-saxonne. Cette domination effective n'est pas perçue comme telle par les acteurs qui font le monde de l'art international, en raison même de la réticularité inscrite dans la contemporanéité. En effet, et qu'il s'agisse du monde de l'art contemporain à une échelle régionale, nationale ou à une échelle internationale, les échanges quotidiens des participants au monde de l'art, les collaborations, les stratégies de réassurance même, se font sur la base effective d'affinités à la fois esthétiques et relationnelles qui construisent, de maillon en maillon, un ensemble de réseaux. Cette réticularité est inscrite dans la contemporanéité pour une autre raison: l'étroitesse de l'audience des diverses formes d'expressions qui sont rattachées à la contemporanéité rend nécessaire la multiplication de réseaux interconnectés et internationaux.

Même si, dans un contexte global de mondialisation des échanges, ces réseaux se ramifient et diffusent toujours plus largement les influences déjà dominantes, l'art contemporain est, du point de vue de son organisation, moins territorial que rhizomatique (Bellavance, 2000). Ainsi, l'art contemporain a bien un territoire, mais il est international et réticulaire et son ancrage est moins géographique qu'affinitaire.

59. Cette observation rejoint celle faite par A. Quemin qui souligne la façon dont certaines manifestations d'art contemporain, pourtant internationales, peuvent être considérées par les experts comme «locales», car ne touchant, par leur audience, qu'un groupe d'États ou un continent. 
Les caractéristiques de la contemporanéité sont à comprendre dans un mouvement plus ample et ancien qui, parallèlement à l'évolution des moyens de communication, tend à substituer aux communautés fondées sur le partage d'un espace et d'une histoire des communautés fondées sur le partage d'intérêts (Wellman et Gulia, 1999). En changeant les modalités par lesquelles les groupes peuvent désormais se constituer en réseaux, les technologies de l'information et de la communication favorisent, au moins potentiellement, la capacité des défenseurs des particularismes locaux à se mobiliser en divers points du monde, comme en atteste l'audience grandissante de l'«antimondialisation». Ainsi, et de façon spéculative, on avancera l'idée suivante: dans ce contexte de mondialisation des échanges et de protestation contre cette mondialisation, la vogue multiculturaliste, conjuguée avec l'affirmation progressive des pays périphériques, pourrait alors donner un nouveau souffle aux expressions identitaires locales et faire émerger un monde de l'art trouvant à s'organiser dans d'autres espaces que celui occupé par l'art contemporain.

\section{RÉSUMÉ}

À travers un siècle de relations entre art et territoire en région Midi-Pyrénées, l'article s'intéresse à la façon dont les acteurs, collectifs et individuels, ont érigé en ressources les notions de territoire et d'identité. Abordée de façon chronologique, la première partie de l'article retrace les périodes au cours desquelles le mouvement régionaliste et les milieux artistiques se sont retrouvés pour porter la revendication d'une identité spécifique. La seconde partie cherche à dépasser les circonstances propres à chacun des moments de l'histoire régionale et dégage les composantes humaines et discursives - les acteurs, le discours identitaire, le « retard» - et les variantes qu'elles enregistrent.

\section{SUMMARY}

In an examination of a century of relations between art and territory in the Midi-Pyrenean region, the present article focuses on ways actors (groups and individuals) have built notions of space and identity into resources. Treated in a chronological manner, the first part of this article traces periods in which the regionalist movement and artistic milieus joined together to bring forth demands for specific identity. The second part of the article tries to go beyond the circumstances of each of these moments of regional history in an analysis of human and discursive components - actors, identity discourses, the "delay" — and variants at different times.

\section{RESUMEN}

A través de un siglo de relaciones entre el arte y el territorio en la región de los Pirineos centrales, el artículo se interesa a la manera en que los actores, colectivos e individuales, erigieron en recursos las nociones de territorio e identidad. Abordado de manera cronológica, la primera parte del artículo recuerda los períodos en el curso de los cuales el movimiento regionalista y los medios artísticos se encontraron para sostener la reivindicación de una identidad específica. La segunda parte busca sobrepasar las circunstancias propias a cada uno de los momentos de la historia regional y despeja los componentes humanos y discursivos - los actores, el discurso identitario, el «retraso»-y las variaciones que ellas registran. 


\section{BIBLIOGRAPHIE}

Azam, M. (1998), Parcours d'artistes ou le talent en questions. La reconnaissance et l'artiste en Midi-Pyrénées, Thèse de sociologie, Université Toulouse Le Mirail.

Bellavance, Guy (2000), Monde et réseaux de l'art. Diffusion, migration et cosmopolitisme en art contemporain, Montréal, Éd. Liber.

Bourdieu, P. (1980), «L'identité et la représentation. Éléments pour une réflexion critique sur l'idée de région", ARSS, novembre, $\mathrm{n}^{\circ} 35$.

Castelnuovo, E. et C. Ginsburg (1980), «Domination symbolique et géographie artistique dans l'histoire de l'art italien", ARSS, novembre, $\mathrm{n}^{\circ} 35$.

Chosson, J.F. (1999), «Un imaginaire citoyen: cultures, territoires communs, création artistique», Enquêtes et témoignages, $\mathrm{n}^{\circ} 163$.

Gras, C. et G. Livet (1977), Régions et régionalisme en France du XVIII siècle à nos jours, Actes publiés, Paris, PUF.

Grossetti, M. (1994), Université et territoire — Un système local d'enseignement supérieur, Toulouse et MidiPyrénées, Grossetti, Toulouse, Presse universitaire du Mirail.

Lafont, R. (1974), La revendication occitane, Paris, Éd. Flammarion, coll. «Histoire vivante».

Lagarde, P. (1977), Clefs pour la régionalisation, Paris, Éd. Seghers, coll. «Clefs».

Meмmi, A. (1997), «Les fluctuations de l'identité culturelle», Esprit, janvier, $n^{\circ} 1$.

Menger, P.M. (1993), «L'hégémonie parisienne. Économie et politique de la gravitation artistique», Paris, Les Annales, $\mathrm{n}^{\circ} 48$.

Mollard, C. (1986), «L'art dans toutes ses régions — Arts plastiques en France», Autrement, Série France, $\mathrm{n}^{\circ} 4$, Paris, mars 1991.

Monnier, G. (1986), Des beaux-arts aux arts plastiques. Une histoire sociale de l'art, Éditions de la Manufacture. Moulin, R. (1992), L'artiste, L'institution, le marché, Paris, Éd. Flammarion.

Poche, B. (1992), Tout art est local, Paris, Éd. L'Harmattan.

Quemin, A. (2001), Le rôle des pays prescripteurs sur le marché et dans le monde de l'art contemporain, rapport au ministère des Affaires étrangères, DGCID, juin.

Rivet, L. (1989), La vie artistique à Toulouse de 1888 à 1939, Thèse d'histoire de l'art, Toulouse, Université de Toulouse Le Mirail.

Vigezzi, M. (2002), «Le renouveau des ensembles économiques régionaux», Communication à la journée d'étude CEDECE «Monde, régions, identités: coexistence, interaction, contradiction», Grenoble II, mars.

Wellman, B. et M. Gulia (1999), «Net Surfers Don’t Ride Alone: Virtual Communities as Communities», «Communities and Cyberspace», New York, edited by P. Kollock and M. Smith, Routledge.

\section{SOURCES}

LePaGe, J., Opus International, juin-juillet 1974, $\mathrm{n}^{\circ} 51$.

Castan, F., La Mostra n ${ }^{\circ} 22,1987$ et n 33/34, 1990.

Labatut, E., Le fRAC Midi-Pyrénées, un exemple de la problématique Paris-province, Mémoire de DESS, 1991. Meyer Himhoff, B., Pictura Edelweiss, nº 2, 1983, p. 103.

Manuel, P., Pictura Edelweiss, $\mathrm{n}^{\circ}$ 5, 1984.

Millau, D., «Misérabilisme de l'art ou la rigueur de l'inertie», Autrement, Série France, nº 4 , Paris, mars 1991. Axe Sud, $\mathrm{n}^{\circ}$ 4/5, 1982.

Chronique de l'Art Vivan, $t$ O6, 1972.

Opus International, $\mathrm{O}_{4}, 1974$.

Eighty, «Créateurs à suivre», 1990.

«Une journée culture en région. Jeudi 31 janvier 2002. Les actes», édité par le Conseil régional Midi-Pyrénées. Actes des troisièmes rencontres nationales des FRAC, Lyon, 10 et 12 mai 1986.

Catalogue de l'exposition «Peinture à Toulouse en 1964». 